\title{
Photo-polymerization and -copolymerization of Aromatic Diacrylates
}

\author{
Chiaki Azuma and Naoya Ogata \\ Department of Chemistry, Sophia University \\ Chiyoda-ku, Tokyo, Japan.
}

(Received October 13, 1972)

\begin{abstract}
Photo-polymerization and-copolymerization of three aromatic diacrylates, i.e., $o-, m$-, and $p$-phenylene diacrylates were investigated in order to elucidate the reactivity of the double bond of $\alpha, \beta$-unsaturated carbonyl compounds toward light. Photopolymerizability of $p$-phenylene diacrylate was highest among these three diacrylates, and it yielded rapidly a cross-linked polymer on irradiation with a high-pressure mercury lamp in tetrahydrofuran solution. Activation energies of photopolymerization of these three diacrylates were much lower than those of thermal polymerization with a radical initiator. Photopolymerization of $o$-phenylene diacrylate-induced cyclopolymerization, yielding a ladder-type polymer with a high inherent-viscosity.

$o$-Phenylene diacrylate and styrene copolymerized and formed a soluble copolymer on irradiation of light.
\end{abstract}

KEY WORDS Photopolymerization / Cyclopolymerization / Aromatic

Diacrylates / Ladder Polymer / $\alpha, \beta$-Unsaturated Carbonyl Compounds /

Photocopolymerization / Styrene /

It was previously found ${ }^{1}$ that $p$-phenylene diacrylate (hydroquinone diacrylate) rapidly polymerized in solution on irradiation with sun light or a high-pressure mercury lamp and solvents had a marked influence on the rate of the photopolymerization. Photopolymerizability of three aromatic diacrylates including $o-, m$-, and $p$ phenylene diacrylate was investigated in order to compare the reactivity of vinyl groups of three aromatic diacrylates toward light, and the results are described in this paper.

Inter- or intra-molecular polymerizations of nonconjugated divinyl compounds have been widely studied by many investigators ${ }^{2-4}$ and such divinyl compounds as diallyl phthalate were known to polymerize to a ladder-type polymer owing to structurally favorable conformation of two vinyl groups in the same molecule. ${ }^{5,6} p$ Phenylene diacrylate polymerized ${ }^{1}$ in solution both by irradiation or by heating with a radical initiator to form an insoluble polymer with a cross-linked structure. However, it is expected that 0 - or $m$-phenylene diacrylates may cyclopolymerize, forming a ladder polymer. Therefore, structural analyses of polymers obtained by photoinduced polymerization of three aromatic diacrylates were also carried out and the polymerization mechanism was discussed.

Kinetics of the photoinduced or thermal copolymerizations of these aromatic diacrylates with styrene were also studied and the reactivities of the double bond of these monomers were discussed in terms of the photosensitivity of $\alpha, \beta$ unsaturated carbonyl compounds.

\section{EXPERIMENTAL}

\section{Materials}

$o-, m$ - or $p$-Phenylene diacrylate (PDA) were synthesized by the reaction of $1 \mathrm{~mol}$ of corresponding dihydric phenols with $2 \mathrm{~mol}$ of acryloyl chloride in tetrahydrofuran (THF) solution in the presence of $2 \mathrm{~mol}$ of triethylamine.

After filtration of triethylamine salt, THF was removed by evaporation. Each of the monomers was completely purified by repeating recrystallizations three or four times from ethanol $/ n$-hexane. $m$-PDA was purified by distillation in vacuo in the presence of cuprous chloride so that no polymerization occurred during the distillation. Each of the monomers was identified by elementary and infrared-spectral analyses. 
Photo-polymerization and -copolymerization of aromatic diacrylates

\begin{tabular}{lcccc}
\hline Monomer & $\mathrm{Mp}$ or $\mathrm{Bp}$ & $\mathrm{C}, \%$ & $\mathrm{H}, \%$ & $\mathrm{O}, \%$ \\
\hline$o$-PDA & $35^{\circ} \mathrm{C}$ & 66.0 & 4.57 & 29.4 \\
$m$-PDA & $109-110^{\circ} \mathrm{C} / 1 \mathrm{mmHg}$ & 65.8 & 4.29 & 29.9 \\
$p$-PDA & $88-89^{\circ} \mathrm{C}$ & 65.1 & 4.59 & 30.3 \\
(Calcd) & - & $(66.0)$ & $(4.63)$ & $(29.4)$ \\
\hline
\end{tabular}

Solvents and styrene were carefully purified by conventional methods before use. Particularly, THF was carefully purified under a nitrogen atmosphere in order to remove peroxide since peroxide might initiate polymerization of PDA.

\section{Polymerization}

Photo-polymerization and-copolymerization. A given amount of each PDA was dissolved in benzene or THF in the concentration of 0.25 $\mathrm{mol} / l$ and the solution was placed in a quartz tube $(2 \times 10 \mathrm{~cm})$ in vacuo. The tube was irradiated at 10,30 or $50^{\circ} \mathrm{C}$ at a distance of $1.5 \mathrm{~cm}$ from

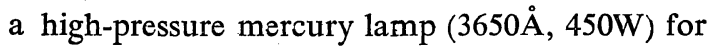
a given period. The distribution of wavelengths of the mercury lamp is shown in Figure 1.

The quantum yield was defined as the overall quantum yield shown in the following equation.

$$
\Phi=\frac{\text { Number of molecules reacted }}{\text { Number of quanta absorbed by sample }}
$$

The photoquantum number was $I_{0}^{i}=8.4 \times 10^{17}$ quanta/sec, which was measured by the ferric potassium oxalate method.
Given amounts of each PDA and styrene were dissolved in benzene or THF in the total monomer concentration of $1.0 \mathrm{~mol} / l$ and the solution was placed in a quartz tube in vacuo. The solution was irradiated at $30^{\circ} \mathrm{C}$ with the mercury lamp previously described.

After the photo-polymerization or -copolymerization, polymers or copolymers were collected by pouring the solution in excess methanol, and purified by repeating dissolution in benzene and reprecipitation with methanol.

Thermal Polymerization and Copolymerization. Radical polymerization and copolymerization of PDA with styrene were carried out at various temperatures in benzene or THF solutions in the presence of azobisisobutyronitrile as an initiator.

After a suitable interval, polymers and copolymers were collected by pouring the solutions in excess of methanol and purified by repeating dissolution in benzene and reprecipitation with methanol. When polymers and copolymers were insoluble in common solvents, they were simply washed with benzene and dried.

\section{Polymer Characterization}

Each of polymers was analyzed by elementary and infrared analyses. The amount of residual unsaturation in polymers was determined by the calibration curve which was obtained by comparing the intensity ratio $\log \left(I_{0} / I\right)_{980} / \log \left(I_{0} / I\right)_{800-690}$ in infrared spectra of polymers with unsaturation

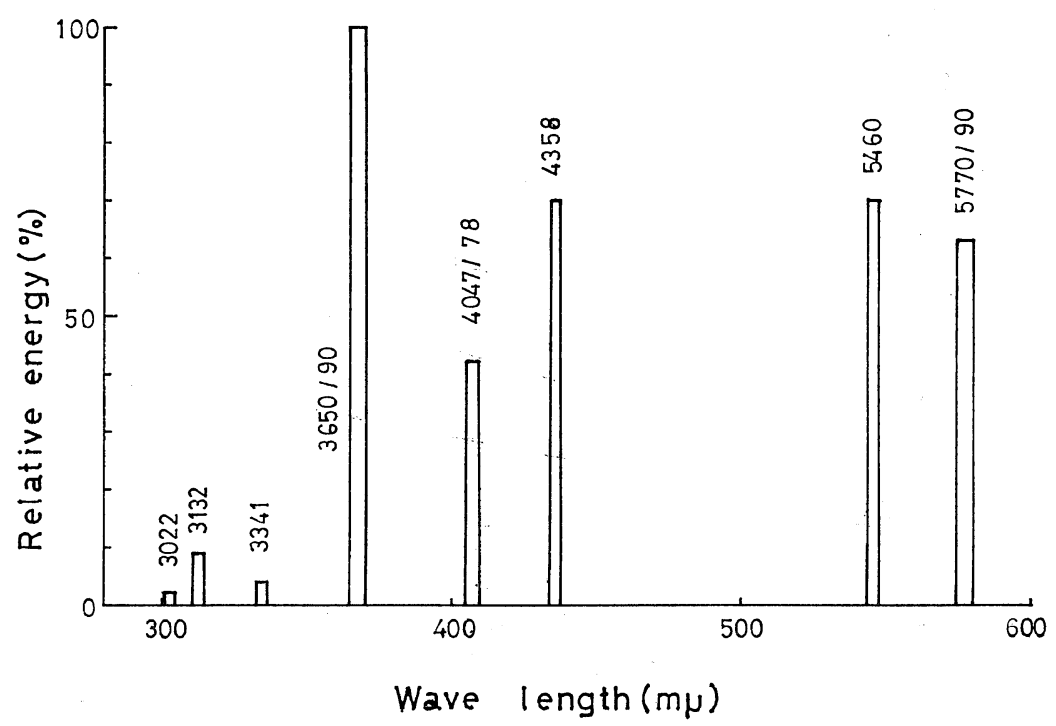

Figure 1. Distribution of wavelength of the high-pressure mercury lamp (450W). 
values of polymers.

Unsaturation values of polymers were measured from the amount of absorbed hydrogen by means of catalytic hydrogenation of polymers which was carried out in methanol under atmospheric hydrogen in the presence of $\mathrm{U}-\mathrm{Ni}$ B catalyst after the saponification of polymers with aqueous $5-\%$ sodium hydroxide solution.

Copolymer compositions were determined from the intensity ratio $\log \left(I_{0} / I\right)_{690-900} / \log \left(I_{0} / I\right)_{700}$ ( $I_{690-900}$ : absorption due to disubstituted benzene, $I_{700}$ : absorption due to monosubstituted benzene) of infrared spectra of copolymers, by using calibration curves which were determined from the elementary analyses of copolymers. The amount of residual unsaturation in copolymers was determined by the method previously described.

\section{RESULTS AND DISCUSSION}

\section{Photopolymerization}

Figure 2 indicates the rate of the photopolymerization of $o-, m-$, or $p$-PDA at $30^{\circ} \mathrm{C}$ in benzene or THF solutions, while Figure 3 shows the rates of the thermal polymerization of these monomers at $60^{\circ} \mathrm{C}$. It is seen in Figure 2 that three PDAs were polymerized by irradiation of light in solution. Solid crystals of these PDAs were not converted into polymers even after a prolonged irradiation.

Apparent overall rates of the photo- and thermal- polymerizations are summarized in Table I.

Photopolymerization of these aromatic diacrylate took place more rapidly in THF than in benzene and the polymerizability of aromatic diacrylates is not arranged in a simple order in THF or benzene solutions.

Ultraviolet spectra of these aromatic diacrylates revealed that these PDAs absorbed light below $360 \mathrm{~m} \mu$, as shown in Figure 4. Therefore, effective wavelength of light for the photoinduced polymerization of these aromatic diacrylates might be below $360 \mathrm{~m} \mu$ although no confirmation was made.

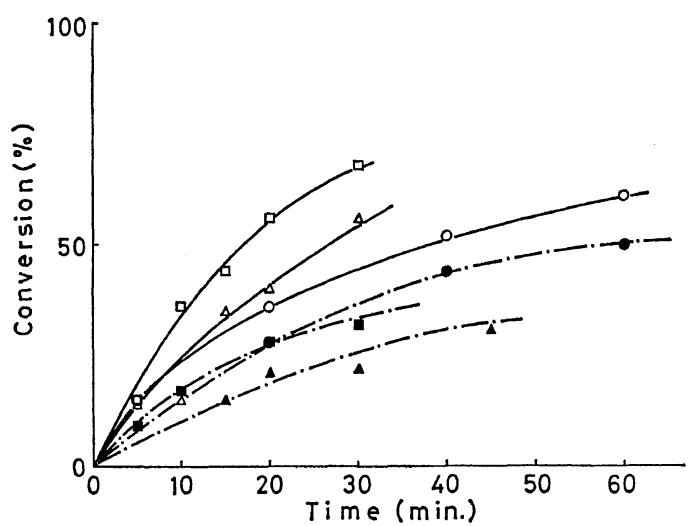

Figure 2. Rates of the photoinduced polymerization of aromatic diacrylates at $30^{\circ} \mathrm{C}$.

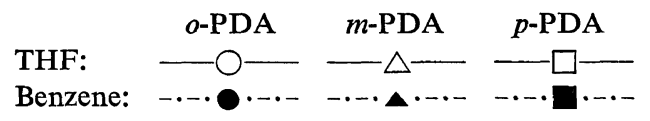

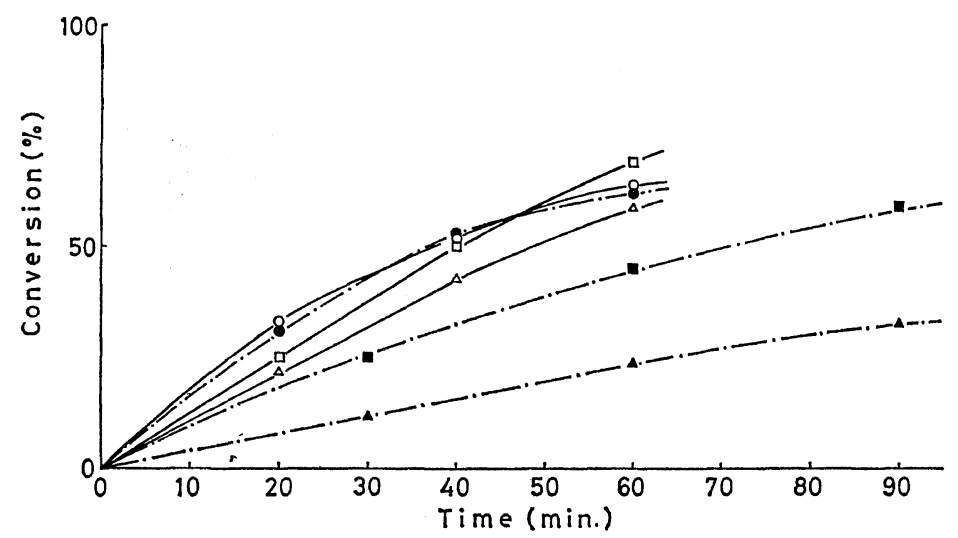

Figure 3. Rates of the thermal polymerization of aromatic diacrylates at $60^{\circ} \mathrm{C}$.

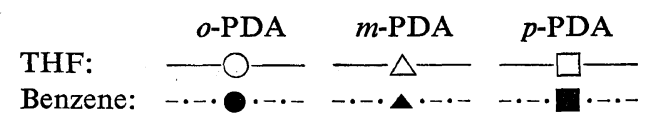


Photo-polymerization and -copolymerization of aromatic diacrylates

Table I. The overall rates and activation energies of the polymerization of PDAs

\begin{tabular}{|c|c|c|c|c|c|c|c|c|}
\hline \multirow{2}{*}{ Monomer } & \multirow{2}{*}{ Solvent } & \multicolumn{4}{|c|}{ Photopolymerization ${ }^{\text {a }}$} & \multicolumn{3}{|c|}{ Thermal polymerization } \\
\hline & & Temp, ${ }^{\circ} \mathrm{C}$ & $R_{\mathrm{p}}$ & $\Delta E$ & $\Phi$ & Temp, ${ }^{\circ} \mathrm{C}$ & $R_{\mathrm{p}}$ & $\Delta E$ \\
\hline \multirow{6}{*}{$o$-PDA } & THF & 10 & 6.3 & & 5.2 & 45 & 1.3 & \\
\hline & & 30 & 10.4 & 4.6 & 8.5 & 60 & 7.5 & 23 \\
\hline & & 50 & 16.7 & & 13.6 & 75 & 27.0 & \\
\hline & Benzene & 10 & 3.3 & & 5.5 & 45 & 1.0 & \\
\hline & & 30 & 7.0 & 7.4 & 11.8 & 60 & 6.9 & 25 \\
\hline & & 50 & 16.7 & & 28.0 & 80 & 53.3 & \\
\hline \multirow{6}{*}{$m$-PDA } & THF & 10 & 7.1 & & 2.3 & 45 & 0.83 & \\
\hline & & 30 & 10.4 & 3.5 & 3.4 & 60 & 4.4 & 20 \\
\hline & & 50 & 15.0 & & 4.9 & 80 & 17.5 & \\
\hline & Benzene & 10 & 3.3 & & 1.1 & 45 & 0.29 & \\
\hline & & 30 & 4.6 & 3.5 & 1.6 & 60 & 1.7 & 26 \\
\hline & & 50 & 6.7 & & 2.2 & 80 & 18.8 & \\
\hline \multirow[t]{6}{*}{$p$-PDA } & THF & 10 & 10.4 & & 2.4 & 45 & 1.5 & \\
\hline & & 30 & 14.6 & 3.0 & 3.4 & 60 & 5.1 & 20 \\
\hline & & 50 & 20.4 & & 4.7 & 80 & 33.3 & \\
\hline & Benzene & 10 & 4.2 & & 2.3 & 45 & 0.42 & \\
\hline & & 30 & 8.3 & 6.0 & 4.5 & 60 & 3.5 & 29 \\
\hline & & 50 & 14.6 & & 8.0 & 80 & 37.0 & \\
\hline
\end{tabular}

${ }^{a} R_{\mathrm{p}}$, overall rate, $(\mathrm{mol} / \mathrm{lsec}) \times 10^{5} ; \Delta E$, activation energy, $\mathrm{kcal} / \mathrm{mol} ; \Phi$, quantum yield, molecules/quanta.

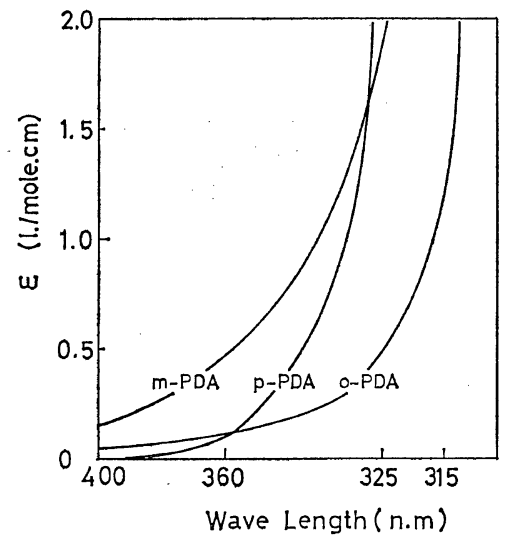

Figure 4. UV spectra of aromatic diacrylates in THF solution; monomer concn, $0.25 \mathrm{~mol} / l$.

Activation energies of photo- or thermal- polymerizations are summarized in Table $I$, where it is seen that activation energies are $1 / 4$ to $1 / 5$ lower than those of the thermal polymerization. Ether solvents such as THF or dioxane polymerized $p$-PDA more rapidly than benzene as previously repeated. ${ }^{1}$ The accelerating effect of ether solvents might be related with electron-donating character of the ether oxygen which might form a kind of charge-transfer complex with PDA in the excited state. However, no evidence for the formation of charge-transfer complex between THF and PDA monomers was found.

The lower activation energies of the photoinduced polymerization than that of the thermal polymerization might be ascribed to the difference in initiation reaction since the photopolymerization of these aromatic diacrylates probably proceeds in radical mechanism in nature as previously reported. ${ }^{1}$ The excitation of molecules by irradiation causes radical formation which requires less energy than the thermal polymerization with a radical initiator, thus resulting in the low activation energy.

Results of the analyses of the polymers obtained at the initial stage of the polymerization are summarized in Table II, where it is seen that a marked difference in polymer character is observed for the polymers obtained from $p$-PDA. The amount of residual unsaturation was remarkably small in the polymers from $o$-PDA when compared with the polymers from $m$ - or $p$-PDA, and the polymers from $o$-PDA were soluble in THF, while the polymers from $m$ - or $p$-PDA were insoluble in any common solvents, indicating the formation of polymers with cross-linked structure as the 


\section{Azuma and N. Ogata}

Table II. The character of polymers

\begin{tabular}{|c|c|c|c|c|c|c|c|c|c|c|c|c|c|}
\hline \multirow{2}{*}{ Monomer } & \multirow{2}{*}{ Solvent } & \multicolumn{6}{|c|}{ Photopolymerization $^{\mathrm{a}}$} & \multicolumn{6}{|c|}{ Thermal polymerization } \\
\hline & & $\underset{{ }^{\circ} \mathrm{C}}{\text { Temp, }}$ & $\underset{\text { min }}{\text { Time }}$ & $\underset{\%}{\text { Yield, }}$ & R.U., & $\eta_{\mathrm{inh}}$ & Solb. & $\underset{{ }^{\circ} \mathrm{C}}{\text { Temp, }}$ & $\underset{\text { min }}{\text { Time }}$ & $\underset{\%}{\text { Yield, }}$ & R.U., & $\eta_{\mathrm{inh}}$ & Solb. \\
\hline \multirow[t]{2}{*}{$o$-PDA } & THF & $\begin{array}{l}10 \\
30 \\
50\end{array}$ & $\begin{array}{l}30 \\
20 \\
10\end{array}$ & $\begin{array}{l}35 \\
36 \\
30\end{array}$ & $\begin{array}{l}13 \\
16 \\
12\end{array}$ & $\left.\begin{array}{l}0.15 \\
0.12 \\
0.12\end{array}\right\}$ & soluble & $\begin{array}{l}45 \\
60 \\
75\end{array}$ & $\begin{array}{r}120 \\
20 \\
10\end{array}$ & $\begin{array}{l}39 \\
33 \\
46\end{array}$ & $\begin{array}{l}13 \\
16 \\
13\end{array}$ & $\left.\begin{array}{l}0.12 \\
0.12 \\
0.12\end{array}\right\}$ & soluble \\
\hline & Benzene & $\begin{array}{l}10 \\
30 \\
50\end{array}$ & $\begin{array}{l}60 \\
20 \\
10\end{array}$ & $\begin{array}{l}28 \\
28 \\
27\end{array}$ & $\begin{array}{r}15 \\
13 \\
9\end{array}$ & $\left.\begin{array}{l}0.94 \\
1.30 \\
1.22\end{array}\right\}$ & swell & $\begin{array}{l}45 \\
60 \\
80\end{array}$ & $\begin{array}{r}180 \\
20 \\
10\end{array}$ & $\begin{array}{l}35 \\
31 \\
76\end{array}$ & $\begin{array}{r}10 \\
7 \\
11\end{array}$ & $\left.\begin{array}{l}3.14 \\
2.36 \\
1.15\end{array}\right\}$ & swell \\
\hline \multirow[t]{2}{*}{$m$-PDA } & THF & $\begin{array}{l}10 \\
30 \\
50\end{array}$ & $\begin{array}{l}30 \\
15 \\
10\end{array}$ & $\begin{array}{l}43 \\
35 \\
36\end{array}$ & $\begin{array}{l}73 \\
39 \\
37\end{array}$ & $\left.\begin{array}{l}0.15 \\
0.12 \\
0.12\end{array}\right\}$ & swell & $\begin{array}{l}45 \\
60 \\
80\end{array}$ & $\begin{array}{r}120 \\
20 \\
10\end{array}$ & $\begin{array}{l}24 \\
22 \\
42\end{array}$ & $\begin{array}{l}64 \\
42 \\
64\end{array}$ & $\left.\begin{array}{l}0.15 \\
0.12 \\
0.15\end{array}\right\}$ & swell \\
\hline & Benzene & $\begin{array}{l}10 \\
30 \\
50\end{array}$ & $\begin{array}{l}40 \\
20 \\
15\end{array}$ & $\begin{array}{l}20 \\
22 \\
22\end{array}$ & $\begin{array}{l}72 \\
29 \\
31\end{array}$ & $\left.\begin{array}{l}0.44 \\
0.40 \\
0.46\end{array}\right\}$ & swell & $\begin{array}{l}45 \\
60 \\
80\end{array}$ & $\begin{array}{r}180 \\
60 \\
20\end{array}$ & $\begin{array}{l}11 \\
24 \\
66\end{array}$ & $\begin{array}{l}41 \\
42 \\
34\end{array}$ & $\left.\begin{array}{l}0.62 \\
0.60 \\
0.35\end{array}\right\}$ & swell \\
\hline \multirow[t]{2}{*}{$p$-PDA } & THF & $\begin{array}{l}10 \\
30 \\
50\end{array}$ & $\begin{array}{l}15 \\
10 \\
10\end{array}$ & $\begin{array}{l}36 \\
36 \\
47\end{array}$ & $\begin{array}{l}54 \\
21 \\
22\end{array}$ & $\left.\begin{array}{l}0.15 \\
0.12 \\
0.12\end{array}\right\}$ & swell & $\begin{array}{l}45 \\
60 \\
80\end{array}$ & $\begin{array}{r}120 \\
20 \\
10\end{array}$ & $\begin{array}{l}44 \\
25 \\
59\end{array}$ & $\begin{array}{l}45 \\
53 \\
54\end{array}$ & $\left.\begin{array}{l}0.12 \\
0.12 \\
0.12\end{array}\right\}$ & swell \\
\hline & Benzene & $\begin{array}{l}10 \\
30 \\
50\end{array}$ & $\begin{array}{l}30 \\
20 \\
15\end{array}$ & $\begin{array}{l}24 \\
28 \\
30\end{array}$ & $\begin{array}{l}54 \\
56 \\
55\end{array}$ & $\left.\begin{array}{l}0.31 \\
0.34 \\
0.42\end{array}\right\}$ & swell & $\begin{array}{l}45 \\
60 \\
80\end{array}$ & $\begin{array}{r}180 \\
30 \\
20\end{array}$ & $\begin{array}{l}17 \\
25 \\
58\end{array}$ & $\begin{array}{l}45 \\
67 \\
55\end{array}$ & $\left.\begin{array}{l}0.96 \\
0.72 \\
0.48\end{array}\right\}$ & swell \\
\hline
\end{tabular}

a R.U., residual unsaturation; $\eta_{\text {inh }}$, in concd $\mathrm{H}_{2} \mathrm{SO}_{4}$ at $30^{\circ} \mathrm{C}$; Solb., solubility in THF.

polymerization proceeded. ${ }^{4}$

Figure 5 shows the relation between the amount of residual unsaturation of the polymers and the polymer yield. The amount of residual unsaturation of the polymer from $o$-PDA was less than $15 \%$, regardless of the polymer yield, while that from $m$ - or $p$-PDA decreased with increasing the polymer yield. The inherent viscosity of resulting polymers from $o$-PDA decreased with temperatures

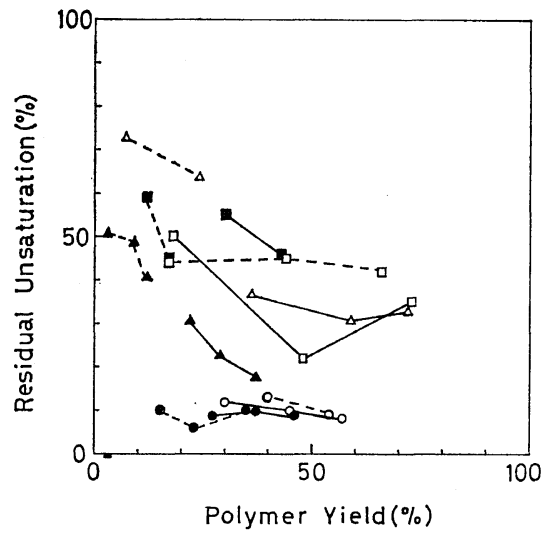

Figure 5. Relations between polymer yield and residual unsaturation.

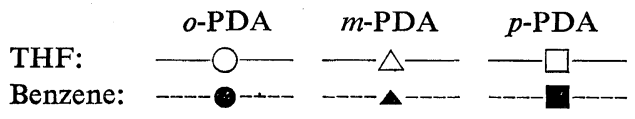

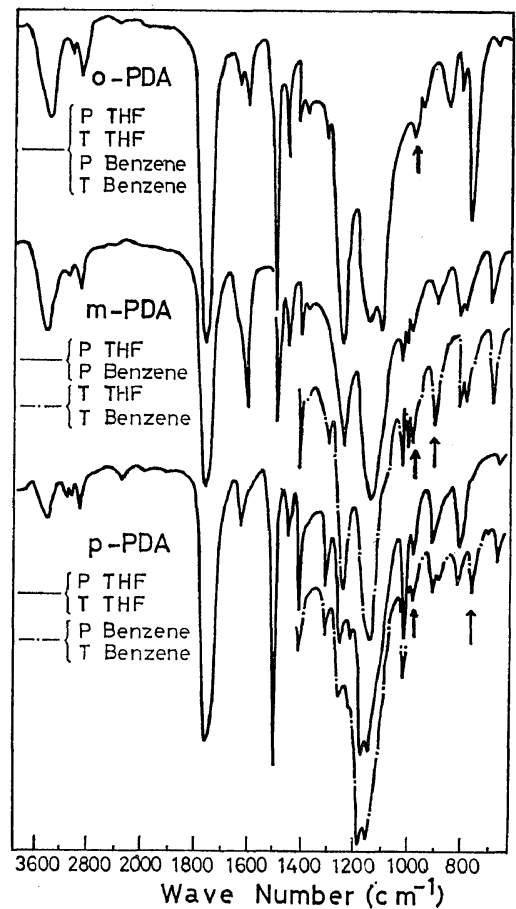

Figure 6. Infrared spectra of polymers from aromatic diacrylates: $\mathrm{P}$, photopolymerization; $\mathrm{T}$, thermal polymerization. 
Photo-polymerization and -copolymerization of aromatic diacrylates

of the polymerization as indicated in Table II and polymers with inherent viscosity as high as 1 to 2 were obtained in concentrated sulfuric acid.

Infrared spectra of the polymers from these aromatic diacrylates are shown in Figure 6, which reveals that absorptions owing to vinyl groups are weak for the polymer from $o$-PDA when compared with polymers from $m$ - or $p$-PDA as indicated by arrows in Figure 6 .
The results described above suggest that a cyclopolymerization might take place to yield a ladder-type polymer in the case of the photoinduced and thermal polymerizations of $o$-PDA, while an intermolecular polymerization favorably proceeds to such a extent that insoluble polymers with a cross-linked structure are formed in the case of the $m$ - or $p$-PDA polymerizations. The polymerization of these aromatic diacrylates can be schematically shown as below

Polymerization of $o-P D A$ :
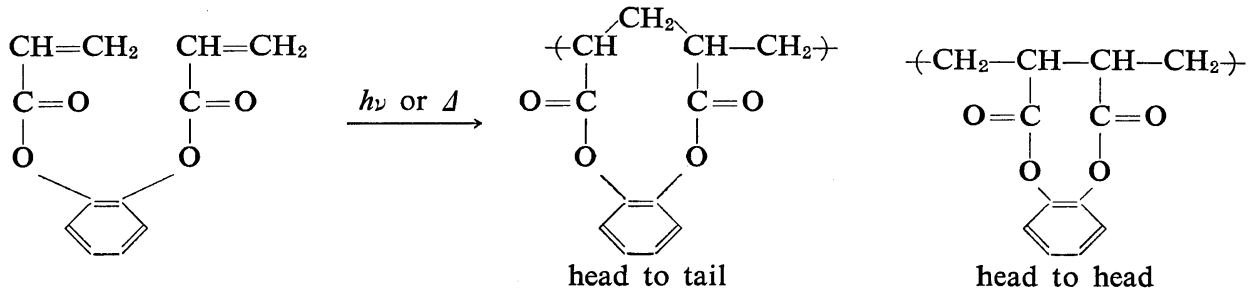

Polymerization of $m$ - or $p$-PDA:

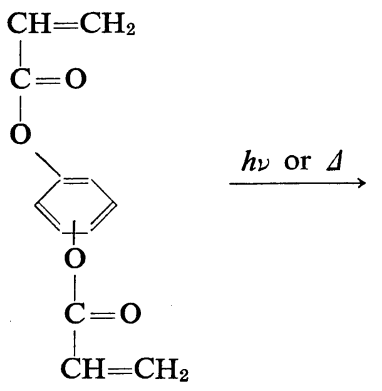<smiles>C=CC(=O)Oc1ccc2cc1OC(=O)C(C)CCOCOC2</smiles>

No further effort was made to determine the exact structure of the polymer from o-PDA whether it has 8 - or 9-membered rings as a result of the cyclopolymerization.

It is presumed that the photopolymerization of these PDAs proceeds in radical mechanism in nature since hydroquinone retarded the polymerization as described in a previous paper. ${ }^{1}$ However, it is suggested that the structure of growing-end radicals of the polymer from $o$-PDA is different from that from $m$ - or $p$-PDA since the inherent viscosity of the polymer from $o$-PDA suffers a marked influence by solvent both in photoinduced and thermal polymerization at the initial stage of the polymerization. The structure of growing-end radicals of the polymer from $o$-PDA might have a cyclic structure so that a solvent effect on the termination reaction might be observed.

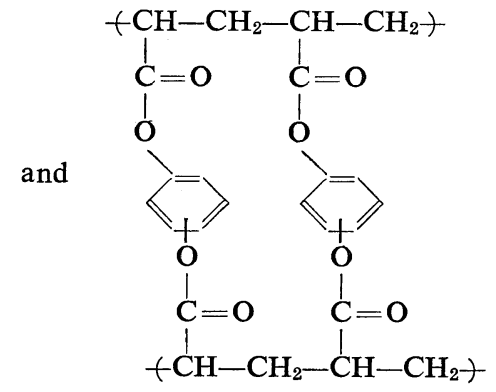

\section{Copolymerization with Styrene}

Rates of the photoinduced copolymerization of three PDAs with styrene at the molar ratio of $1 / 1$ in the feed are shown in Figure 7 . When $m$ - or $p$-PDA solutions containing styrene were irradiated with a high-pressure mercury lamp, whole solutions became a highly swollen gel within $30 \mathrm{~min}$ and copolymers insoluble in any common solvents were obtained.

Copolymerization of $o$-PDA with styrene proceeded in a homogeneous solution without formation of gel, and copolymers soluble in THF or benzene were obtained. Infrared spectra of these soluble or insoluble copolymers indicated the existence of carbonyl group and monosubstituted benzene, which suggest that copolymerization of these PDAs with styrene had occurred.

The apparent overall rates of copolymerization are summarized in Table III, which indicates 


\section{Azuma and N. Ogata}

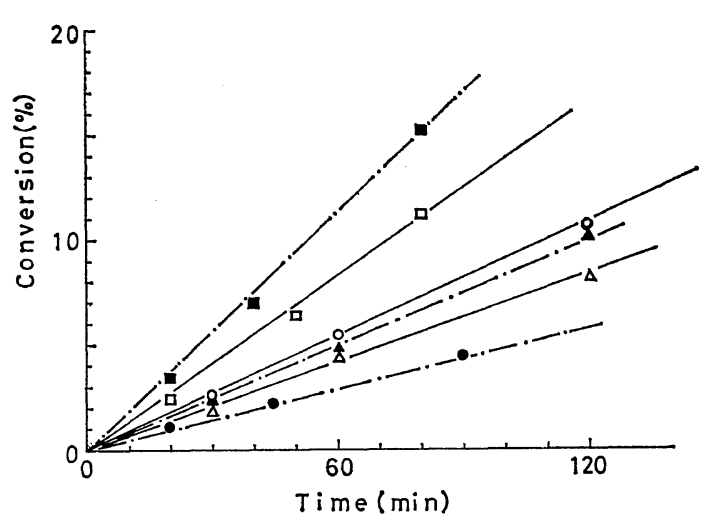

Figure 7. Rates of the photoinduced copolymerization of aromatic diacrylates with styrene at $30^{\circ} \mathrm{C}$.

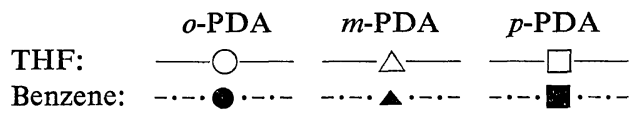

that $p$-PDA copolymerized most rapidly with styrene among these three PDAs by the irradiation of light, and the orders of copolymerization rates in both THF and benzene are as follows: $p$-PDA $\gg o$-PDA, $m$-PDA.

Copolymer composition curves together with unsaturation values of copolymers are shown in Figures 8-10. Unsaturation values of copolymers from $m$-PDA were as high as $100 \%$ (when one of the two vinyl groups reacted and the other was left unreacted, it was defined as $100 \%$ of unsaturation value of copolymers) in the range of higher molar ratios of PDA in the monomer feed and then they decreased with increasing amount of styrene in the feed. These results suggest that copolymerization of $m$ - or $p$-PDA occurred, forming copolymers with pendant acrylate groups on the main chain in higher molar ratios of PDA in the feed and then the pendant acrylate groups reacted with styrene to form cross-linked copolymers when the amount of styrene in the monomer feed increased.

On the other hand, unsaturation values of the copolymer from $o$-PDA were quite low, regardless of the monomer molar ratios in the feed. The copolymers from $o$-PDA and styrene were soluble in common organic solvents including benzene or THF in the whole range of molar ratios in the monomer feed. Therefore, it is presumed that cyclopolymerization of o-PDA might take place to form soluble copolymers with a ladder-type structure and no intermolecular reaction occurs to yield cross-linked copolymers.

The propagation of the copolymerization of PDA with styrene can be expressed in the following equations when the intramolecular cyclization of PDA and the reaction of residual unsaturated groups of copolymers are ignored

$$
\begin{aligned}
& \mathbf{M}_{1} \cdot+\mathbf{M}_{1} \stackrel{k_{11}}{\longrightarrow} \mathrm{M}_{1} \cdot \\
& \mathbf{M}_{1} \cdot+\mathrm{M}_{2} \stackrel{k_{12}}{\longrightarrow} \mathrm{M}_{2} \cdot \\
& \mathbf{M}_{2} \cdot+\mathrm{M}_{2} \stackrel{k_{22}}{\longrightarrow} \mathrm{M}_{2} .
\end{aligned}
$$

\begin{tabular}{|c|c|c|c|c|c|c|c|}
\hline \multirow{2}{*}{$\mathbf{M}_{1}$} & \multirow{2}{*}{ Solvent } & \multirow{2}{*}{ System $^{2}$} & \multicolumn{5}{|c|}{ 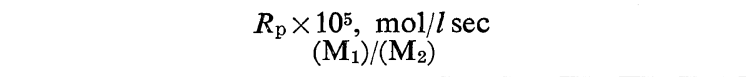 } \\
\hline & & & $1 / 9$ & $3 / 7$ & $5 / 5$ & $7 / 3$ & $9 / 1$ \\
\hline \multirow[t]{4}{*}{$o$-PDA } & THF & $\mathbf{P}$ & 0.70 & 1.4 & 1.7 & 2.2 & 3.9 \\
\hline & & $\mathrm{T}$ & 0.70 & 1.4 & 2.2 & 3.0 & 5.3 \\
\hline & Benzene & $\mathbf{P}$ & 0.31 & 0.83 & 0.86 & 1.4 & 3.9 \\
\hline & & $\mathrm{T}$ & 1.1 & 2.2 & 4.2 & 6.7 & 16 \\
\hline \multirow[t]{4}{*}{$m$-PDA } & THF & $\mathbf{P}$ & 0.72 & 1.1 & 1.3 & 1.5 & 2.5 \\
\hline & & $T$ & 0.72 & 1.9 & 2.1 & 2.5 & 3.1 \\
\hline & Benzene & $\mathbf{P}$ & 0.47 & 0.89 & 1.4 & 1.7 & 2.7 \\
\hline & & $T$ & 0.94 & 4.0 & 5.5 & 8.0 & 17 \\
\hline \multirow[t]{4}{*}{$p$-PDA } & THF & $\mathrm{P}$ & 0.22 & 2.2 & 2.8 & 5.4 & 7.9 \\
\hline & & $\mathrm{T}$ & 1.5 & 5.0 & 10 & 14 & 17 \\
\hline & Benzene & $\mathbf{P}$ & 0.84 & 2.2 & 3.2 & 5.2 & 8.0 \\
\hline & & $T$ & 1.4 & 3.8 & 5.8 & 7.8 & 10 \\
\hline
\end{tabular}

Table III. The apparent overall rates of copolymerization

a $\mathrm{P}$, photopolymerization at $30^{\circ} \mathrm{C} ; \mathrm{T}$, thermal polymerization at $60^{\circ} \mathrm{C}$. 
Photo-polymerization and -copolymerization of aromatic diacrylates

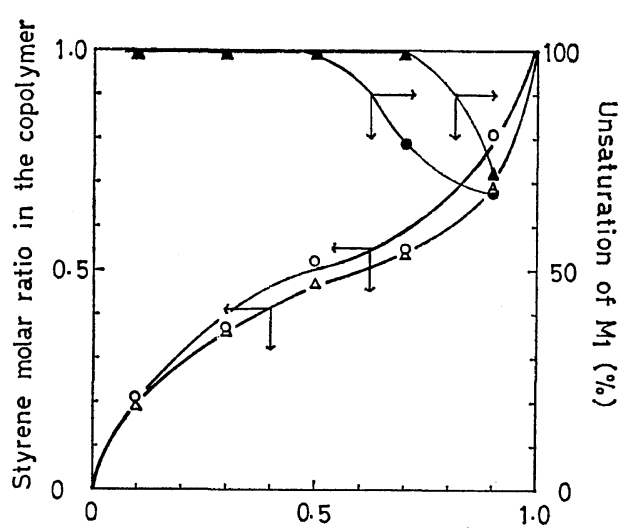

Styrene(M2) molar ratio in the feed

Figure 8. Copolymer composition curve and unsaturation value of $o$-PDA $\left(M_{1}\right)$ with styrene $\left(M_{2}\right)$ in the photopolymerization at $30^{\circ} \mathrm{C}$ or thermal polymerization at $60^{\circ} \mathrm{C}$ in THF: $\bigcirc, \bullet$, photopolymerization; $\triangle, \mathbf{\Delta}$, thermal polymerization.

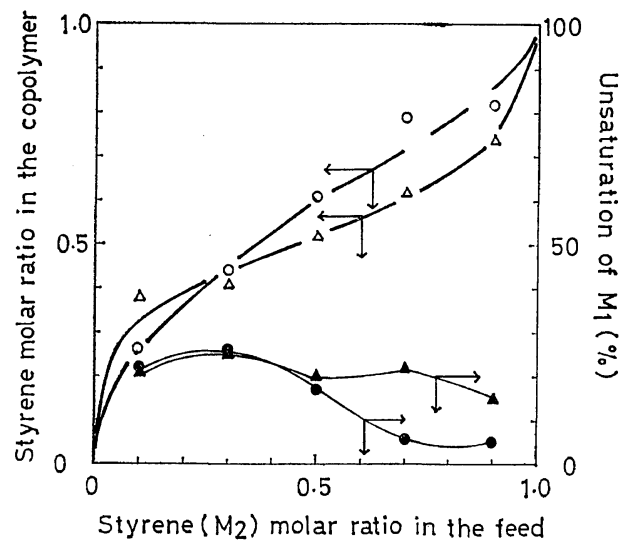

Figure 9. Copolymer composition curve and unsaturation value of $m$-PDA $\left(\mathrm{M}_{1}\right)$ with styrene $\left(\mathbf{M}_{2}\right)$ in the photopolymerization at $30^{\circ} \mathrm{C}$ or thermal polymerization at $60^{\circ} \mathrm{C}$ in THF: $\bigcirc, \bullet$, photopolymerization; $\Delta, \mathbf{\Lambda}$, thermal polymerization.

$$
\mathrm{M}_{2} \cdot+\mathrm{M}_{1} \stackrel{k_{21}}{\longrightarrow} \mathrm{M}_{1} \cdot
$$

where $M_{1}$ and $M_{2}$ denote PDA and styrene, respectively. Apparent monomer reactivity ratios of these aromatic diacrylate can be calculated according to the ordinary Mayo-Lewis equation for radical copolymerization. However, the intramolecular cyclization actually occurred during the polymerization of PDAs. Smets $^{7}$ and Aso $^{8}$ have derived copolymer composition equa-

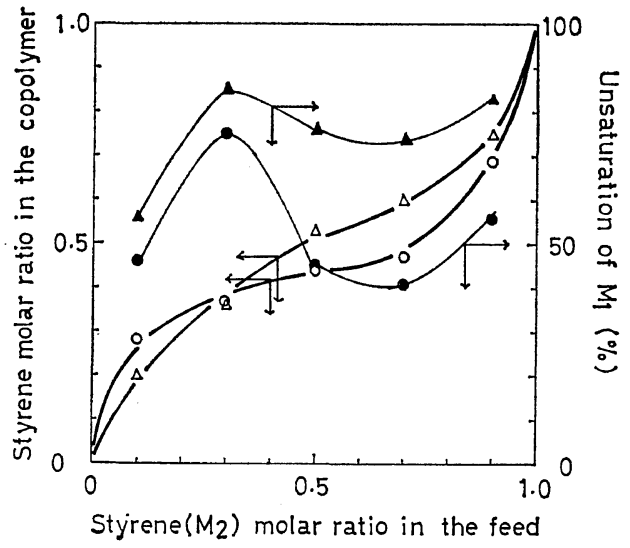

Figure 10. Copolymer composition curve and unsaturation value of $p$-PDA $\left(\mathrm{M}_{1}\right)$ with styrene $\left(\mathrm{M}_{2}\right)$ in the photopolymerization at $30^{\circ} \mathrm{C}$ or thermal polymerization at $60^{\circ} \mathrm{C}$ in THF: $O, \bullet$, photopolymerization; $\triangle, \mathbf{\Delta}$, thermal polymerization.

tions in the case of the copolymerization accompanied with cyclization reaction. When divinyl monomer radical $\mathbf{M}_{1}$. reacts with monomers to cause the intramolecular cyclization reaction together with simple monomer additions to $M_{1} \cdot$, the following reactions should be taken into account

$$
\begin{aligned}
\mathrm{M}_{1} \cdot \stackrel{k_{\mathrm{c}}}{\longrightarrow} \mathrm{m} . \\
\mathrm{m} \cdot+\mathrm{M}_{1} \stackrel{k_{31}}{\longrightarrow} \mathrm{M}_{1} . \\
\mathrm{m} \cdot+\mathrm{M}_{2} \stackrel{k_{32}}{\longrightarrow} \mathrm{M}_{2} .
\end{aligned}
$$

where $\mathrm{m}$ denotes the cyclic radical formed by the intramolecular cyclization reaction. Then, the copolymer composition equation is derived by assuming the steady state of these seven propagation reactions of eq $1-7$ as follows

$$
\begin{aligned}
& \frac{\mathrm{d}\left[\mathrm{M}_{1}\right]}{\mathrm{d}\left[\mathrm{M}_{2}\right]} \\
& =\frac{2 k_{11}\left[\mathrm{M}_{1} \cdot\right]\left[\mathrm{M}_{1}\right]+2 k_{21}\left[\mathrm{M}_{2} \cdot\right]\left[\mathrm{M}_{1}\right]+2 k_{31}[\mathrm{~m} \cdot]\left[\mathrm{M}_{1}\right]}{k_{12}\left[\mathrm{M}_{1} \cdot\right]\left[\mathrm{M}_{2}\right]+k_{22}\left[\mathrm{M}_{2} \cdot\right]\left[\mathrm{M}_{2}\right]+k_{32}[\mathrm{~m} \cdot]\left[\mathrm{M}_{2}\right]} \\
& =\frac{2\left[\mathrm{M}_{1}\right]}{\left[\mathrm{M}_{2}\right]} \frac{2 r_{1}\left[\mathrm{M}_{1}\right]+\left[\mathrm{M}_{2}\right]+K_{\mathrm{c}}{ }^{\prime}}{r_{2}\left[\mathrm{M}_{2}\right]+2\left[\mathrm{M}_{1}\right]+K_{\mathrm{c}}{ }^{\prime}\left\{\frac{r_{2}\left[\mathrm{M}_{2}\right]+2\left[\mathrm{M}_{1}\right]}{2 r_{3}\left[\mathrm{M}_{1}\right]+\left[\mathrm{M}_{2}\right]}\right\}} \\
& \quad-\frac{\mathrm{d}\left[\mathrm{M}_{1}\right]}{\mathrm{d}[\mathrm{m}]}=1+\frac{2\left[\mathrm{M}_{1}\right]}{K_{\mathrm{c}}}+\frac{\left[\mathrm{M}_{2}\right]}{K_{\mathrm{c}}{ }^{\prime}}
\end{aligned}
$$




\section{Azuma and N. Ogata}

Table IV. The monomer reactivity ratios and $Q, e$ values

\begin{tabular}{|c|c|c|c|c|c|c|c|c|}
\hline $\mathbf{M}_{1}$ & Solvent & System ${ }^{a}$ & $r_{1}$ & $r_{2}$ & $r_{3}$ & $K_{\mathrm{c}}, \mathrm{mol} / l$ & $Q$ & $e$ \\
\hline \multirow[t]{4}{*}{$o-\mathrm{PDA}$} & \multirow[t]{2}{*}{ THF } & $\mathbf{P}$ & $0.43 \pm 0.22$ & $1.30 \pm 0.70$ & $0.05 \pm 0.15$ & $6.0 \pm 2.0$ & 0.42 & -0.04 \\
\hline & & $\mathbf{T}$ & $0.48 \pm 0.19$ & $0.55 \pm 0.15$ & $0.06 \pm 0.04$ & $8.0 \pm 3.0$ & 0.74 & 0.37 \\
\hline & \multirow[t]{2}{*}{ Benzene } & $\mathbf{P}$ & $0.43 \pm 0.09$ & $0.85 \pm 0.05$ & $0.09 \pm 0.01$ & $7.7 \pm 0.9$ & 0.53 & 0.20 \\
\hline & & $\mathbf{T}$ & $0.45 \pm 0.19$ & $0.42 \pm 0.28$ & $0.16 \pm 0.06$ & $10 \pm 3.0$ & 0.83 & 0.49 \\
\hline \multirow[t]{4}{*}{$m-\mathrm{PDA}^{\mathrm{b}}$} & \multirow[t]{2}{*}{ THF } & $\mathbf{P}$ & $0.12 \pm 0.10$ & $0.49 \pm 0.30$ & & & 0.53 & 0.88 \\
\hline & & $\mathrm{T}$ & $0.17 \pm 0.02$ & $0.33 \pm 0.06$ & & & 0.78 & 0.90 \\
\hline & \multirow[t]{2}{*}{ Benzene } & $\mathbf{P}$ & $0.17 \pm 0.05$ & $0.73 \pm 0.07$ & & & 0.44 & 0.65 \\
\hline & & $\mathbf{T}$ & $0.15 \pm 0.04$ & $0.67 \pm 0.11$ & & & 0.46 & 0.72 \\
\hline \multirow[t]{4}{*}{$p-\mathrm{PDA}^{c}$} & \multirow[t]{2}{*}{ THF } & $\mathbf{P}$ & $0.39 \pm 0.16$ & $0.08 \pm 0.08$ & & & 2.90 & 1.07 \\
\hline & & $\mathbf{T}$ & $0.21 \pm 0.07$ & $0.35 \pm 0.06$ & & & 0.79 & 0.82 \\
\hline & \multirow[t]{2}{*}{ Benzene } & $\mathbf{P}$ & $0.35 \pm 0.06$ & $0.19 \pm 0.19$ & & & 1.40 & 0.85 \\
\hline & & $\mathrm{T}$ & $0.35 \pm 0.09$ & $0.30 \pm 0.08$ & & & 1.00 & 0.70 \\
\hline
\end{tabular}

s $\mathrm{P}$, photopolymerization at $30^{\circ} \mathrm{C} ; \mathrm{T}$, thermal polymerization at $60^{\circ} \mathrm{C} ; \mathrm{M}_{2}$, styrene.

b $r_{3}=0, K_{\mathrm{c}}=0$.

c Reactivity of vinyl group in polymer was regarded as equal to that of monomer and $r_{3}=0, K_{\mathrm{c}}=0$.

$$
\begin{aligned}
-\frac{\mathrm{d}\left[\mathrm{M}_{2}\right]}{\mathrm{d}[\mathrm{m}]}= & {\left[\mathrm{M}_{2}\right]\left\{1+r_{2} \frac{\left[\mathrm{M}_{2}\right]}{2\left[\mathrm{M}_{1}\right]}\right\} } \\
& \times\left\{\frac{1}{K_{\mathrm{c}}{ }^{\prime}}+\frac{1}{2 r_{3}\left[\mathrm{M}_{1}\right]+\left[\mathrm{M}_{2}\right]}\right\}
\end{aligned}
$$

where $r_{1}=k_{11} / k_{12}, \quad r_{2}=k_{22} / k_{21}, \quad r_{3}=k_{31} / k_{11}, \quad K_{\mathrm{c}}{ }^{\prime}=$ $k_{\mathrm{c}} / k_{12}$.

When $-\mathrm{d}\left[\mathrm{M}_{1}\right] / \mathrm{d}[\mathrm{m}]=f_{1}$, eq 10 can be rewritten as follows

$$
\frac{2\left[\mathrm{M}_{1}\right]}{f_{1}-1}=K_{\mathrm{c}}-\frac{1}{r_{1}} \frac{\left[\mathrm{M}_{2}\right]}{f_{1}-1}
$$

By plotting $2\left[\mathrm{M}_{1}\right] /\left(f_{1}-1\right)$ against $\left[\mathrm{M}_{2}\right] /\left(f_{1}-1\right)$, $K_{\mathrm{c}}, K_{\mathrm{c}}{ }^{\prime}$, and $r_{1}$ can be obtained since $f_{1}$ is the reciprocal of the cyclization degree.

When $-\mathrm{d}\left[\mathrm{M}_{2}\right] / \mathrm{d}[\mathrm{m}]=f_{2}$ and $\left[\mathrm{M}_{2}\right] / 2\left[\mathrm{M}_{1}\right]=F$, eq 10 can be rewritten as eq 12

$$
r_{2}=\frac{f_{2}}{F^{2}} \frac{K_{\mathrm{c}}{ }^{\prime}\left(r_{3}+F\right)}{K_{\mathrm{c}}{ }^{\prime}+2\left[\mathrm{M}_{1}\right]\left(r_{3}+F\right)}-\frac{1}{F}
$$

According to eq 12, $r_{2}$ and $r_{3}$ can be obtained by using the intersection method with $K_{\mathrm{c}}{ }^{\prime}=r_{1} K_{\mathrm{c}}$ and these results are summarized in Table IV. Values of $r_{1}$ are almost equal, regardless of the type of copolymerization and solvents, and they are greater than the apparent values of $r_{1}$ calculated according to the Mayo-Lewis equation without respect of cyclization reaction.

Values of $r_{3}$ (the ratio of rate constants of the reaction of cyclic radical with monomers) are smaller than $r_{1}$ and this difference in reactivities of o-PDA radical and its cyclic radical, is possibly due to the steric effect of cyclic radical. Values of $Q$ and $e$ of $o$-PDA are generally smaller than those of $m$ - or $p$-PDA and $r_{2}$ is greater than $r_{1}$ in the $o$-PDA copolymerization. These results suggest that ortho effect causes decrease in reactivity and resonance stability of $o-P D A$ radical.

Values of $e$ of these PDAs are positive as is expected from the electron-accepting character of carbonyl group attached with double bond. The $e$ values in THF solution are larger than those in benzene solution, which suggest that an interaction of propagating radicals with solvent might stabilize the excited state of monomers, resulting in the high reactivity of PDAs in THF.

\section{REFERENCES}

1. N. Ogata and C. Azuma, J. Polym. Sci., Part A-1, 8, 1759 (1971).

2. C. Aso, et al., ibid., 34, 475 (1959).

3. M. Gordon, et al., ibid., 21, 27, 39, 57, 75 (1956).

4. C. Aso, Yukigosei Kagaku Kyokaishi, 21, 678 (1963).

5. W. Simpson, et al., Proc. Roy. Soc., Ser. A, 238, 154 (1956).

6. M. Oiwa, et al., Nihon Kagaku Zasshi (J. Chem. Soc. Japan, Pure Chem. Sect.), 79, 1506 (1958), ibid., 76, 508 (1955).

7. G. Smets, et al., Makromol. Chem., 60, 89 (1963).

8. C. Aso, et al., Kogyo Kagaku Zasshi (J. Chem. Soc. Japan, Ind. Chem. Sect.) 68, 549 (1965). 